\title{
TOXICIDADE DE FILTRADOS FÚNGICOS A Meloidogyne incognita*
}

\section{MAURO J.N. COSTA ${ }^{1}$, VICENTE P. CAMPOS ${ }^{1 * *}$, LUDWIG H. PFENNING ${ }^{1}$, DENILSON F. OLIVEIRA ${ }^{2}$}

\author{
${ }^{1}$ Departamento de Fitopatologia, ${ }^{2}$ Departamento de Química, Universidade Federal de Lavras, \\ Cx. Postal 37, CEP 37.200-000, Lavras, MG.
}

(Aceito para publicação em 20/08/2001)

Autor para correspondência: Vicente Paulo Campos

COSTA, M.J.N, CAMPOS, V.P., PFENNING, L.H. \& OLIVEIRA, D.F. Toxicidade de filtrados fúngicos a Meloidogyne incognita. Fitopatologia Brasileira 26:749-755. 2001.

\section{RESUMO}

Visando à obtenção de novas moléculas nematicidas, 18 filtrados de espécies fúngicas e extratos de sete micélios fúngicos foram estudados in vitro quanto aos efeitos sobre a eclosão, motilidade e mortalidade de juvenis do segundo estádio (J2) de Meloidogyne incognita. Filtrados fúngicos foram obtidos de culturas em meio Czapek. Metanol foi usado como solvente para obter extratos dos micélios fúngicos. Filtrados de Paecilomyces lilacinus, Fusarium moniliforme e Fusarium oxysporum reduziram a motilidade e eclosão, e aumentaram a mortalidade $(\mathrm{P}<0,05)$ de J2 de $M$. incognita de forma semelhante à observada com Aldicarbe. Alguns filtrados fúngicos reduziram a motilidade, mas não causaram a morte de J2. Já os filtrados de
Aspergillus flavus, Cylindrocarpon magnusianum, Fusarium solani e Mortierella sp. reduziram apenas a eclosão de J2. Não houve correlação entre a produção de metabólitos tóxicos e a quantidade de micélio fúngico produzido. No caso específico de $P$. lilacinus, observou-se que após o primeiro dia de cultivo o filtrado fúngico correspondente já apresentava toxidez contra $M$. incognita. Com o aumento do número de dias de cultivo foi elevado o efeito do filtrado sobre o nematóide, sendo que apenas após 13 dias a mortalidade de J2 alcançou o índice de $100 \%$.

Palavras-chave adicionais: eclosão, fitonematóides, Fusarium oxysporum, metabólitos tóxicos, Paecilomyces lilacinus.

\section{ABSTRACT \\ Fungus filtrates toxicity to Meloidogyne incognita}

In the search for new nematicidal molecules, 18 fungal species filtrates and seven fungus mycelium extracts were studied in vitro to see their influence on hatching, mobility and mortality of second stage juveniles (J2) of Meloidogyne incognita. Fungal filtrates were obtained from fifteen day Czapek cultures. Methanol was used as solvent to obtain fungus mycelium extracts. Paecilomyces lilacinus, Fusarium moniliforme and Fusarium oxysporum filtrates reduced $\mathrm{J} 2$ motility and hatching, and increased $\mathrm{J} 2$ mortality
$(\mathrm{P}<0,05)$ similarly to Aldicarb. Some fungus filtrates reduced J2 mobility without leading to death. Aspergillus flavus, Cylindrocarpon magnusianum, Fusarium solani and Mortierella sp. filtrates reduced $(\mathrm{P}<0,05)$ only $\mathrm{J} 2$ hatching. Production of toxic fungus metabolites was not dependent on the amount of mycelium produced. P. lilacinus produced toxic filtrates after one day culturing. Since then, toxicity increased steadily, reaching $100 \% \mathrm{~J} 2$ mortality on the thirteenth day.

\section{INTRODUÇÃO}

Nos últimos anos a sociedade tem priorizado aspectos ambientais, direcionando muitas pesquisas para a descoberta de novas substâncias bioativas que possam ser empregadas no manejo integrado de pragas e doenças, com menos efeitos negativos sobre o meio ambiente (Castro, 1989). Conseqüentemente, vários são os trabalhos que buscam tais substâncias nos fungos que perfazem um grande número de espécies

\footnotetext{
* Parte da dissertação de Mestrado do primeiro autor. Universidade Federal de Lavras. Suporte Financeiro do CNPq.

**Bolsista do CNPq.
}

com potencial para a produção de metabólitos com diversas atividades biológicas (Porter \& Fox, 1993).

No caso específico de fitonematóides, sabe-se que os principais inimigos naturais são fungos, que podem atuar de várias formas (Barron 1977; Naves \& Campos, 1991). Alguns são endoparasitas que penetram no corpo do nematóide através de aberturas naturais ou mesmo pela cutícula (Aschner \& Kohn, 1958); outros, como Paecilomyces lilacinus Bainer e Verticillium chlamydosporium Goddard, parasitas de ovos e de cistos (Mankau, 1979; Jatala et al., 1980); há também os predadores (Arthrobotrys spp., Dactylaria spp., Dactylella spp. e Monacrosporium spp.) que desenvolvem um 
mecanismo de formação de armadilha, por meio do qual a hifa adere ao nematóide para penetrar no seu corpo em seguida (Dijksterhius, 1993). No entanto, o emprego de tais fungos diretamente para o controle de fitonematóides, no campo, não apresentou ainda os resultados conseguidos em laboratório. O potencial em ágar-água destes fungos não representa as condições encontradas nos solos, com seus complexos habitats. Assim, vem surgindo como alternativa o uso de metabólitos fúngicos. Pleurotus ostreatus (Jacq.) Fr., por exemplo, gera minúsculas gotas de toxinas que imobilizam o nematóide em menos de 30 segundos (Barron $\&$ Thorn, 1987). Com P. lilacinus, observou-se que seus metabólitos são capazes de paralizar o desenvolvimento embrionar de Meloidogyne hapla Chitwood, causando morte de $88 \%$ dos embriões (Fitters et al.,1993).

Embora ocorra no Brasil grande diversidade de espécies de fungos, compondo a micobiota do solo, ainda são escassos os estudos sobre o efeito de substâncias que interagem entre os componentes desse ecossistema. Desta forma, objetivou-se neste trabalho, estudar o efeito de filtrados e extratos de micélios fúngicos na eclosão, motilidade e mortalidade de juvenis do segundo estádio (J2) de Meloidogyne incognita (Kofoid \& White) Chitwood.

\section{MATERIAL E MÉTODOS}

\section{Isolamento de fungos}

No isolamento de fungos predadores de nematóides utilizou-se a centrifugação diferencial descrita por Barron (1969) e por Nicolay \& Sikora (1988), adaptada por Naves \& Campos (1991), utilizando-se, para o crescimento de fungos, o meio ágar-água (AA) $2 \%$ e como nematóide isca, Panagrellus redivivus Goodey. Os conídios produzidos foram transferidos para placas de Petri contendo o meio batatadextrose-ágar (BDA). Os fungos isolados foram Arthrobotrys conoides Dreschsler, A. oligospora Fress., A. superba Corda e Monacrosporium doedycoides Drechsler.

Fungos de solos foram obtidos por meio da técnica de placas de Warcup (Warcup, 1950), fazendo-se espalhamento de $1 \mathrm{mg}$ de solo sobre o meio malte-ágar (MA), em placas de Petri estéreis, contendo o antibiótico cloranfenicol (50 ppm). As colônias formadas foram repicadas para novas placas contendo o meio sintético sólido pobre em nutriente (SNA). Obtiveram-se assim, Aspergillus flavus Link: Fr., V. chlamydosporium, Cunninghamella elegans Lendner, Fusarium moniliforme Shelden (isolados 2, 4 e 25), Penicillium Subgen. Furcatum Pitt, Mortierella sp. e Trichoderma viridae Pers. Ex Gray.

Os fungos Cylindrocarpon magnusianum (Sacc.) Wollenw., Fusarium oxysporum Schlecht, Fusarium solani (Mart.) Appel \& Wollenw. Emend. Snyd. \& Hans, P. lilacinus (isolados 01 e 02) e Paecilomyces variotii Bainer foram obtidos de fêmeas de $M$. incognita. Para isto as fêmeas foram retiradas de galhas de raízes de café (Coffea arabica L.) e de tomate (Lycopersicon esculentum Mill.), colocadas em placa de Petri contendo água destilada e esterilizada e em seguida transferidas para placas contendo o meio AA, acrescido do antibiótico cloranfenicol. As colônias fúngicas crescidas sobre essas fêmeas foram repicadas para o meio BDA. Os fungos Sclerotinia sclerotiorum (Lib.) de Bary e Coniothyrium sporulosum (W. Gams \& Domsch) van der Aa, usados no ensaio, faziam parte da coleção de culturas puras do Laboratório de Nematologia do Departamento de Fitopatologia da Universidade Federal de Lavras.

$\mathrm{Na}$ identificação das espécies fúngicas, utilizaramse chaves de identificação atualizadas (Domsch, et al., 1993; Klich \& Pitt, 1984; Liu \& Zhang, 1994; Nelson, et al., 1983; Pitt, 1988; Rubner, 1996).

\section{Obtenção de filtrados e de extratos de micélios fúngicos}

Três discos de $5 \mathrm{~mm}$ de diâmetro das culturas fúngicas crescidas em meio aveia-ágar $(\mathrm{OA})$ foram transferidos para Erlenmeyers de $250 \mathrm{ml}$, contendo $100 \mathrm{ml}$ de meio Czapek. Após 15 dias a $25^{\circ} \mathrm{C}$, em agitador orbital "shaker", a 160 rpm, todo o conteúdo dos Erlenmeyers foi filtrado duas vezes em papel Whatman $\mathrm{n}^{\circ} 1 \mathrm{e}$ em membrana Durapore de $0,22 \mu \mathrm{m}$ de abertura e de $4,7 \mathrm{~mm}$ de diâmetro. O líquido obtido de cada cultura, denominado filtrado fúngico, foi empregado em testes in vitro.

Para a obtenção dos extratos metanólicos, $40 \mathrm{~g}$ de cada micélio e $120 \mathrm{ml}$ de metanol foram colocados em um balão de $250 \mathrm{ml}$ com fundo redondo e junta esmerilhada. Acoplaram-se condensadores de refluxo ao balão e as misturas foram refluxadas por 15 min através da utilização de mantas de aquecimento. Em seguida, as misturas foram filtradas em filtros de vidro sinterizado. Concentraram-se as soluções em evaporador rotatório, sob pressão reduzida, a 35 ${ }^{\circ} \mathrm{C}$, até a remoção total do solvente, o que deu origem a resíduos, que foram separadamente dissolvidos em $1.000 \mathrm{ml}$ de solução do surfactante não iônico Tween 80 a 1\% (v/v) em água destilada, para serem submetidos aos testes in vitro.

As soluções obtidas foram armazenadas em câmara fria a $4{ }^{\circ} \mathrm{C}$ até o momento da sua utilização.

\section{Produção de inóculo de Meloidogyne incognita}

A partir de cultura pura de $M$. incognita, caracterizada através dos aspectos morfológicos da região perineal das fêmeas, conforme descrito por Taylor \& Sasser (1978), e mantida em tomateiros em casa de vegetação, obteve-se uma suspensão de ovos utilizando-se a técnica de Hussey \& Barker (1973), modificada por Boneti \& Ferraz (1981). Os ovos obtidos foram submetidos a duas etapas de desinfestação superficial. 1) A suspensão de ovos foi submetida à técnica de Coolen \& D'Herde (1972), através da qual os ovos foram separados de resíduos de raízes e de outras impurezas. Três alíquotas de $1 \mathrm{ml}$ foram retiradas e colocadas em caixa plástica de contagem e levadas ao microscópio estereoscópio para a contagem de ovos. 2) Em câmara de fluxo laminar, toda a suspensão de ovos foi passada três vezes em água destilada e esterilizada, em peneiras esterilizadas de $0,025 \mathrm{~mm}$, e guardada em câmara fria a $8{ }^{\circ} \mathrm{C}$, até o momento do estabelecimento dos ensaios. 
Toxicidade de filtrados fúngicos a Meloidogyne incognita

Para a obtenção de juvenis foi utilizada uma câmara de eclosão, formada com tela e papel de espessura fina colocados num béquer esterilizado. Montou-se a câmara de eclosão sem aeração, para evitar contaminações.

Efeitos de filtrados e de extratos fúngicos na eclosão, motilidade e na mortalidade de juvenis de Meloidogyne incognita

Dez ml de filtrados ou de extratos fúngicos obtidos como descrito foram colocados em placas de Petri estéreis, de $4,5 \mathrm{~cm}$ de diâmetro. A seguir, foi pipetado $1 \mathrm{ml} \mathrm{de}$ suspensão contendo 1.000 ovos de $M$. incognita e a mistura final foi incubada por 15 dias, a $25^{\circ} \mathrm{C}$, em sala climatizada. Após a incubação foi realizada a avaliação da percentagem de J2 eclodidos. Para o teste de motilidade, $1 \mathrm{ml}$ de suspensão contendo $200 \mathrm{~J} 2$ de $M$. incognita foi colocado em placas de Petri de 4,5 cm de diâmetro. Em seguida, adicionaram-se $10 \mathrm{ml}$ de filtrado ou extrato fúngico, e cada placa foi vedada com película Rolopack. Após $12 \mathrm{~h}$, foi avaliado o número de $\mathrm{J} 2$ aparentemente inativos. Foram considerados inativos os nematóides que apresentavam o corpo com aspecto retilíneo, sob efeito de filtrados e de extratos; ou retorcido, o que ocorria quando os J2 eram submetidos ao Aldicarbe. A seguir, os $\mathrm{J} 2$ foram transferidos para tela de $0,025 \mathrm{~mm}$, substituindo-se o filtrado por água de torneira. Espécimens que permaneceram inativados após $12 \mathrm{~h}$, em água, foram considerados mortos. Cada placa constituiu uma unidade experimental, sendo que foram empregadas seis repetições, sob um delineamento experimental inteiramente casualizado. Constituíram-se tratamentos os filtrados fúngicos ou extratos (Tabela 1), além de três testemunhas: $10 \mathrm{ml}$ de água destilada em substituição aos filtrados ou extratos; $10 \mathrm{ml}$ de meio de cultura Czapek ou do surfactante Tween a 1\%;10 ml de Aldicarbe (50 ppm).

\section{Exposição de ovos e juvenis de Meloidogyne incognita a filtrados fúngicos}

Empregaram-se filtrados de $A$. conoides, $F$. solani e $F$. moniliforme isolado 2, produzidos como descrito. Os ovos e J2 foram produzidos e desinfestados como descrito. Empregou-se apenas uma concentração do filtrado, isto é, sem diluição a partir da original. No estabelecimento do ensaio seguiu-se a mesma metodologia descrita, sendo que a avaliação do ensaio foi feita contando os $\mathrm{J} 2$ eclodidos, a cada $24 \mathrm{~h}$, até 15 dias, enquanto a motilidade de J2 foi avaliada de 30 em $30 \mathrm{~min}$, até $36 \mathrm{~h}$, sem interrupção. O delineamento experimental foi inteiramente casualizado, com cinco repetições.

\section{Incubação de Paecilomyces lilacinus para produção de filtrado}

O fungo foi cultivado em meio BDA e repicado para 30 frascos Erlenmeyer contendo o meio Czapek, para produção de metabólitos secundários, conforme descrito. A cada $24 \mathrm{~h}$, retirou-se um frasco do agitador orbital para que o seu conteúdo fosse submetido à filtração, sendo o filtrado obtido armazenado a $2{ }^{\circ} \mathrm{C}$. Após o término dos frascos, montou-se o ensaio empregando-se como testemunhas água, meio Czapek e Aldicarbe. A avaliação do ensaio foi feita como descrito, sendo o delineamento experimental inteiramente casualizado, com seis repetições.

Efeito da diluição de filtrados fúngicos na eclosão, motilidade e mortalidade de juvenis de Meloidogyne incognita

Os filtrados fúngicos foram diluídos com água destilada e esterilizada, obtendo-se assim soluções 1:0, 1:1, 1:2 e 1:3 (filtrado:água). Em seguida, essas dosagens foram colocadas em contato com os ovos ou J2. Constituíram-se tratamentos os filtrados de $C$. elegans, $F$. moniliforme e de $P$. lilacnius, em ambas as diluições. $\mathrm{O}$ delineamento experimental foi inteiramente casualizado, com seis repetições.

\section{Análise de variância}

Os resultados obtidos foram submetidos à análise de variância e efetuados testes de médias, como o de Skott \& Knott (1974). Em alguns, tais resultados foram ajustados a curvas, por meio de equações estimadas que apresentaram coeficientes de determinação apropriados, confirmados pelos valores de $\mathrm{R}^{2}$.

\section{RESULTADOS E DISCUSSÃO}

Efeito de filtrados fúngicos na eclosão, motilidade e mortalidade de juvenis do segundo estádio (J2) de Meloidogyne incognita

Com diferentes intensidades, todos os filtrados fúngicos apresentaram atividade tóxica contra $M$. incognita, sendo que o melhor resultado foi obtido para o proveniente de F. oxysporum. Para tal filtrado obtiveram-se $98 \%$ de J2 imóveis, 98\% de J2 mortos e apenas 3\% de eclosão de J2 (Tabela 1). Especificamente no teste de motilidade, em todos os casos foram obtidos resultados superiores ao da água, sendo vários filtrados com valores próximos ao do observado para a solução de Aldicarbe. No caso de P. lilacinus, os números estão em pleno acordo com aqueles relatados por Cayrol et al. (1989), segundo os quais o filtrado de tal fungo proporciona $100 \%$ de imobilização de Meloidogyne arenaria (Neal) Chitwood. No teste de mortalidade, excetuando-se os filtrados de $F$. moniliforme isolado $02, F$. oxysporum e $P$. lilacinus isolados 01 e 02 , que apresentaram valores altos e semelhantes aos da motilidade, observa-se que a tendência é ter percentagens de J2 mortos inferiores a de J2 imobilizados. Também vale mencionar que os filtrados dos quatro fungos acima provocaram mortalidade superior à observada para Aldicarbe, e que o proveniente de $F$. moniliforme isolado 25 apresentou valor idêntico ao desse nematicida comercial. Um dos resultados mais negativos foi obtido com o filtrado de F. solani ( $9 \%$ de J2 mortos), o que é condizente com os dados da literatura, que mostra a necessidade de um tempo de exposição bem maior ao filtrado desse fungo para que se possa obter resultados mais 
TABELA 1 - Efeito de filtrados de culturas fúngicas, do nematicida Aldicarbe, meio Czapek sem crescimento fúngico e água, na motilidade, mortalidade e eclosão de juvenis do segundo estádio (J2) de Meloidogyne incognita

\begin{tabular}{|c|c|c|c|c|c|}
\hline Tratamento & pH do filtrado & Peso micélio (g) & J2 imóvel (\%) & $\mathrm{J} 2$ morto (\%) & Eclosão (\%) \\
\hline \multicolumn{6}{|c|}{ Ascomycetes mitospóricos (Hyphomycetes) } \\
\hline Arthrobotrys conoides & 7,39 & 0,28 & $17 \mathrm{~h}$ & $8 \mathrm{n}$ & $72 \mathrm{p}$ \\
\hline Arthrobotrys oligospora & 7,73 & 0,30 & $41 \mathrm{f}$ & $56 \mathrm{~g}$ & $69 \mathrm{n}$ \\
\hline Arthrobotrys superba & 7,60 & 0,40 & $8 \mathrm{i}$ & 141 & $78 \mathrm{r}$ \\
\hline Aspergillus flavus & 8,39 & 0,90 & $98 \mathrm{a}$ & $55 \mathrm{~g}$ & $35 \mathrm{~g}$ \\
\hline Cylindrocarpon magnusianum & 6,08 & 0,94 & $97 \mathrm{~b}$ & $49 \mathrm{~h}$ & $40 \mathrm{~h}$ \\
\hline Fusarium moniliforme isolado 2 & 6,25 & 0,64 & $97 \mathrm{~b}$ & $96 \mathrm{c}$ & $10 \mathrm{c}$ \\
\hline Fusarium moniliforme isolado 4 & 6,06 & 1,40 & $92 \mathrm{c}$ & $20 \mathrm{k}$ & $9 \mathrm{~b}$ \\
\hline Fusarium moniliforme isolado 25 & 5,26 & 1,60 & 98 a & $90 \mathrm{~d}$ & $12 \mathrm{~d}$ \\
\hline Fusarium oxysporum & 5,06 & 1,60 & $98 \mathrm{a}$ & $98 \mathrm{~b}$ & $3 \mathrm{a}$ \\
\hline Fusarium solani & 4,81 & 3,70 & $97 \mathrm{~b}$ & $9 \mathrm{~m}$ & $42 \mathrm{i}$ \\
\hline Monacrosporium doedycoides & 5,05 & 0,28 & $7 \mathrm{i}$ & $6 \mathrm{q}$ & 70 o \\
\hline Paecilomyces lilacinus isolado 01 & 5,21 & 3,16 & 99 a & $99 \mathrm{a}$ & $40 \mathrm{~h}$ \\
\hline Paecilomyces lilacinus isolado 02 & 5,62 & 2,10 & $97 \mathrm{~b}$ & $99 \mathrm{a}$ & $43 \mathrm{i}$ \\
\hline Paecilomyces variotii & 6,56 & 1,50 & $97 \mathrm{~b}$ & $42 \mathrm{i}$ & $76 \mathrm{q}$ \\
\hline Penicillium Subgen. Furcatum & 3,96 & 2,60 & $37 \mathrm{~g}$ & $10 \mathrm{~m}$ & $58 \mathrm{k}$ \\
\hline Trichoderma viride & 7,69 & 0,70 & $83 \mathrm{e}$ & $8 \mathrm{n}$ & $86 \mathrm{~s}$ \\
\hline Verticillium chlamydosporium & 6,13 & 1,30 & $94 \mathrm{c}$ & $35 \mathrm{j}$ & $76 \mathrm{q}$ \\
\hline \multicolumn{6}{|c|}{ Ascomiceto mitospórico (Coelomycetes) } \\
\hline Coniothyrium sporulosum & 7,12 & 2,33 & $8 \mathrm{i}$ & $5 \mathrm{p}$ & $67 \mathrm{~m}$ \\
\hline \multicolumn{6}{|l|}{ Ascomycota } \\
\hline Sclerotinia sclerotiorum & 4,83 & 1,91 & $89 \mathrm{~d}$ & $48 \mathrm{~h}$ & $50 \mathrm{j}$ \\
\hline \multicolumn{6}{|l|}{ Zygomycota (Mucorales) } \\
\hline Cunninghamella elegans & 7,46 & 1,62 & 99 a & 84 e & 591 \\
\hline Mortierella sp. & 5,76 & 1,96 & 98 a & $63 \mathrm{f}$ & $32 \mathrm{f}$ \\
\hline Testemunha 1 (água) & - & - & $2 \mathrm{k}$ & $2 \mathrm{q}$ & $89 \mathrm{t}$ \\
\hline Testemunha 2 (meio Czapek) & - & - & $3 \mathrm{j}$ & $5 \mathrm{p}$ & $85 \mathrm{~s}$ \\
\hline Testemunha 3 (Aldicarbe) & - & - & $94 \mathrm{c}$ & $90 \mathrm{~d}$ & $14 \mathrm{e}$ \\
\hline
\end{tabular}

Médias seguidas da mesma letra, nas colunas, não diferem entre si, pelo teste de Scott \& Knott (1974), a 5\% de probabilidade.

expressivos (Mani \& Sethi, 1984). No teste de eclosão o grande destaque foi o filtrado de $F$. oxysporum, só permitindo que $3 \%$ do ovos tivessem $\mathrm{J} 2$ eclodidos, enquanto que com Aldicarbe se obteve $14 \%$ de eclosão. Os filtrados de $F$. moniliforme isolado 2, 4 e 25 também se mostraram promissores, com apenas 10, 9 e $12 \%$ de eclosão de J2 (Tabela 1).

Vale observar que fungos conhecidamente como predadores de nematóides como A. conoides, A. superba e M. doedycoides, produziram filtrados com baixa toxicidade a $M$. incognita, o que indica ineficácia na secreção de metabólitos tóxicos a fitonematóides. Talvez, para tais fungos as estruturas de captura de nematóides, como anéis constritivos, anéis não constritivos ou hifas adesivas, tenham maior importância do que a produção de metabólitos tóxicos.

\section{Exposição de ovos e juvenis de Meloidogyne incognita a filtrados fúngicos}

No teste de motilidade, o melhor resultado foi obtido com o filtrado de $F$. moniliforme, que afetou em torno de $60 \%$ do total de $\mathrm{J} 2 \mathrm{em}$ apenas $30 \mathrm{~min}$ (Figura 1). Com 1,5 h de exposição a percentagem de $\mathrm{J} 2$ imóveis já estava próxima de $100 \%$. Com o filtrado de $F$. solani, a ação foi mais lenta, sendo necessárias de 3 a 4 h de exposição para que se alcançasse valores próximos de $100 \%$ de imobilização de
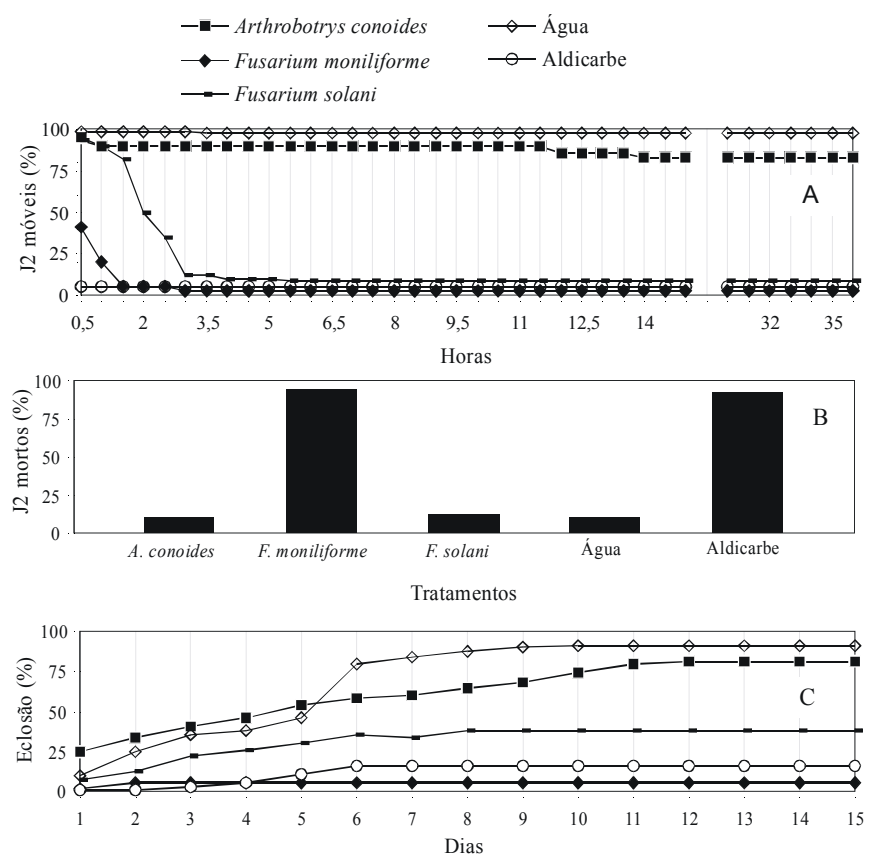

FIG. 1 - Efeito de filtrados fúngicos na motilidade (A) e na mortalidade (B) avaliados 36 h após o contato; e na eclosão (C) de juvenis do segundo estádio (J2) de Meloidogyne incognita. 
J2. Quanto ao filtrado de $A$. conoides, mesmo após várias horas o número de $\mathrm{J} 2$ móveis continuou bastante elevado. Depois de $36 \mathrm{~h}$ expostos aos filtrados observou-se que, em todos os casos, o número de $\mathrm{J} 2$ mortos era aproximadamente idêntico ao de nematóides imóveis: $10 \%$ com $A$. conoides, $10 \%$ com $F$. solani e $97 \%$ com $F$. moniliforme. No teste de eclosão, $F$. moniliforme manteve o número de $\mathrm{J} 2$ eclodidos próximo de zero, mesmo após 15 dias, resultado ligeiramente superior ao observado com solução de Aldicarbe. O filtrado de $F$. solani permitiu o aumento gradativo do número de eclosões até o $8^{\circ}$ dia, quando estabilizou em $40 \%$ de $\mathrm{J} 2$ eclodidos. O filtrado de $A$. conoides teve o pior desempenho, com uma curva bem próxima àquela obtida com água (Figura $1)$.

\section{Incubação de Paecilomyces lilacinus para a produção de filtrado}

Com o teste de motilidade observou-se que após o $1^{\circ}$ dia já eram produzidos metabólitos tóxicos a $M$. incognita (Figura 2). Em torno do $6^{\circ}$ dia houve considerável aumento em tal produção, com o número de $\mathrm{J} 2$ móveis aproximandose de zero e assim permanecendo até o $30^{\circ}$ dia. No teste de mortalidade também se observou efeito após o $1^{\circ}$ dia de cultivo. Porém, nesse caso o aumento da produção de substâncias tóxicas se deu de forma bem mais suave, alcançando valores próximos de $100 \%$ de $\mathrm{J} 2$ mortos no $13^{\circ}$ dia. Talvez essa diferença em relação ao observado no teste de motilidade se deva à produção de mais de uma substância tóxica a $M$. incognita por parte do fungo. No teste de eclosão, o aumento na produção de substâncias tóxicas a $M$. incognita foi bem menor e mais suave do que o observado nos testes de motilidade e mortalidade. O mínimo de J2 eclodidos foi alcançado com o filtrado relativo ao cultivo de $P$. lilacinus por 20 dias, o que coincide com a maior produção de micélio. Após tal período começa a haver ligeira redução na eficiência dos filtrados, o que talvez seja decorrente da metabolização das substâncias ativas pelo fungo. Esse resultado sugere uma maior sensibilidade aos metabólitos fúngicos por parte dos nematóides na fase de $\mathrm{J} 2$ eclodidos do que na fase de multiplicação celular da formação do embrião. Outra possibilidade consiste na produção de diferentes metabólitos por parte do fungo. De qualquer forma, este resultado está de acordo com o trabalho realizado por Fitters et al. (1993) que estudaram o efeito de
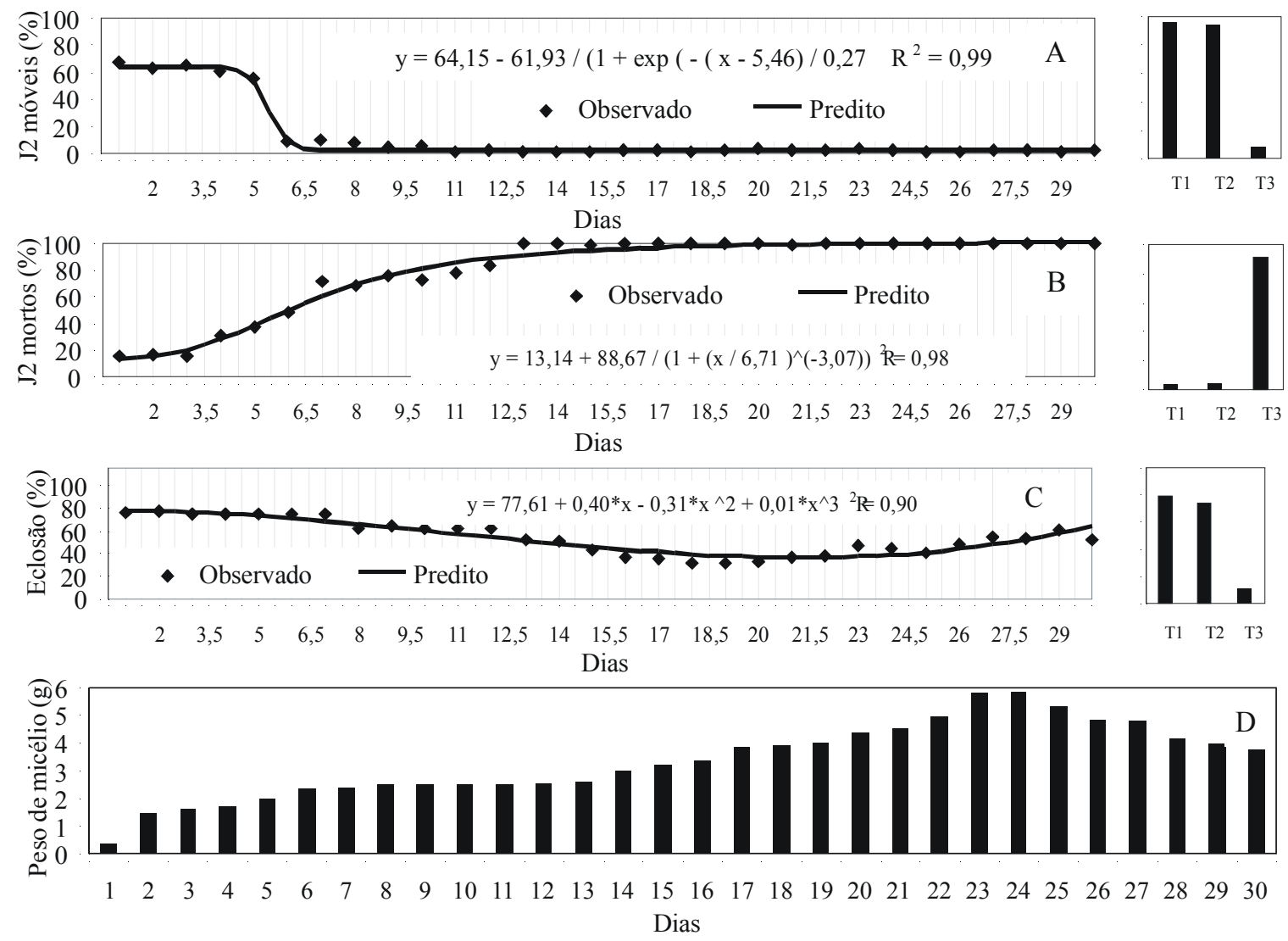

FIG. 2 - Efeito de filtrados obtidos de culturas de Paecilomyces lilacinus cultivadas por até 30 dias em meio Czapek, na motilidade (A), mortalidade (B) e na eclosão (C) de juvenis do segundo estádio (J2) de Meloidogyne incognita. Peso de micélio fúngico no período (D). Testemunhas: 1) incubação dos J2 em água (T1); 2) em meio Czapek (T2); 3) no nematicida Aldicarbe (T3). 
filtrados desse mesmo fungo após o $15^{\circ}$ dia de cultivo na embriogênese de ovos de Meloidogyne hapla. Naqueles ovos tratados com os filtrados, o desenvolvimento do embrião parou após dois - quatro dias e ocorreram $88 \%$ de mortes embrionares.

Efeito da diluição de filtrados fúngicos na eclosão, motilidade e mortalidade de juvenis de Meloidogyne incognita

Ao se trabalhar com diluições dos filtrados de $C$. elegans, F. moniliforme isolado 02 e $P$. lilacinus isolado 01, observou-se que, até 1:3 (filtrado:água), apenas aquele correspondente ao primeiro fungo apresentou aumento significativo no número de $\mathrm{J} 2$ móveis. Isso indica que nos outros dois filtrados as substâncias ativas estão presentes em maiores concentrações ou são mais eficientes (Figura 3). No teste de mortalidade a diluição dos filtrados de $C$. elegans e de $F$. moniliforme diminuiu consideravelmente o número de $\mathrm{J} 2$ mortos. No caso de $P$. lilacinus, as variações foram relativamente pequenas até à diluição $1: 2$ (filtrado :água). No entanto, ao passar de 1:2 para 1:3 (filtrado:água), houve uma queda na percentagem de J2 mortos, que passou de aproximadamente $95 \%$ para $30 \%$. Quanto ao teste de eclosão, observou-se que todos os filtrados apresentaram aumentos nos números de $\mathrm{J} 2$ eclodidos, porém de forma intermediária. Ou seja, a variação não foi tão alta quanto no teste de mortalidade, nem tão baixa quanto no de motilidade. Extratos obtidos a partir de micélios fúngicos pela
extração com metanol

Fazendo uso do processo de extração com metanol, observou-se que os micélios fúngicos também possuem substâncias tóxicas a $M$. incognita, sendo os extratos de $M$. doedycoides superiores àqueles obtidos com os filtrados correspondentes. Já com os outros fungos, os extratos sempre apresentaram resultados semelhantes ou inferiores aos dos filtrados (Tabela 2). Vale mencionar que a produção de metabólito tóxico a fitonematóide não depende diretamente da quantidade de micélio que o fungo produz. $F$. solani e $P$. Subgen. Furcatum produziram grande massa micelial, entretanto, não sintetizaram substâncias com razoável toxicidade a J2. Por outro lado, F. moniliforme isolados 2 e 25 produziram pouca massa micelial, porém excretaram no meio de cultura metabólitos altamente tóxicos a J2 de $M$. incognita (Tabela 1).

TABELA 2 - Efeito de extratos obtidos de micélio fúngico, extraídos com metanol e, do nematicida Aldicarbe, na motilidade, mortalidade e eclosão de juvenis do segundo estádio (J2) de Meloidogyne incognita

\begin{tabular}{|c|c|c|c|c|c|}
\hline \multirow{2}{*}{\multicolumn{2}{|c|}{$\begin{array}{l}\text { Tratamento } \\
\text { Ascomycetes mitospóricos (Hyphomycetes) }\end{array}$}} & $\begin{array}{c}\text { J2 imóvel } \\
(\%)\end{array}$ & $\begin{array}{c}\text { J2 morto } \\
(\%)\end{array}$ & \multicolumn{2}{|c|}{$\begin{array}{c}\text { Eclosão } \\
(\%)\end{array}$} \\
\hline & & & & & \\
\hline Arthrobotrys conoides & 5 & $\mathrm{f}$ & $6 \mathrm{f}$ & 85 & f \\
\hline Arthrobotrys oligospora & 32 & $\mathrm{~d}$ & $60 \mathrm{c}$ & 42 & $\mathrm{~b}$ \\
\hline Arthrobotrys superba & 2 & $\mathrm{~g}$ & $5 \mathrm{f}$ & 79 & $\mathrm{e}$ \\
\hline Fusarium moniliforme isolado 2 & 43 & $\mathrm{c}$ & $50 \mathrm{~d}$ & 42 & $\mathrm{~b}$ \\
\hline Fusarium solani & 7 & $\mathrm{e}$ & $16 \mathrm{e}$ & 80 & e \\
\hline Monacrosporium doedycoides & 79 & $\mathrm{~b}$ & $70 \mathrm{~b}$ & 62 & $\mathrm{~d}$ \\
\hline Paecilomyces lilacinus isolado 01 & 34 & $-d$ & $40 \mathrm{~d}$ & - -46 & c \\
\hline Testemunha 1 (água) & 1 & $\mathrm{~g}$ & $3 \mathrm{f}$ & 90 & $\mathrm{~g}$ \\
\hline Testemunha 2 (Aldicarbe) & 93 & $\mathrm{a}$ & $88 \quad \mathrm{a}$ & 15 & $\mathrm{a}$ \\
\hline
\end{tabular}

Médias seguidas da mesma letra, nas colunas, não diferem entre si, pelo teste de Scott \& Knott (1974) a 5\% de probabilidade
A

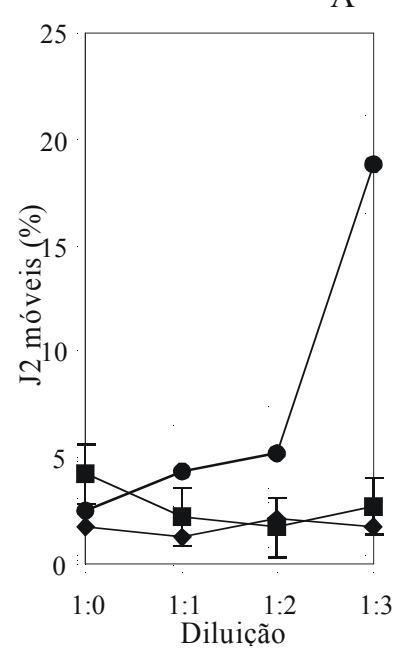

- Cunninghamella elegans

$\rightarrow$ - Fusarium moniliforme

$\longrightarrow$ Paecilomyces lilacinus

B

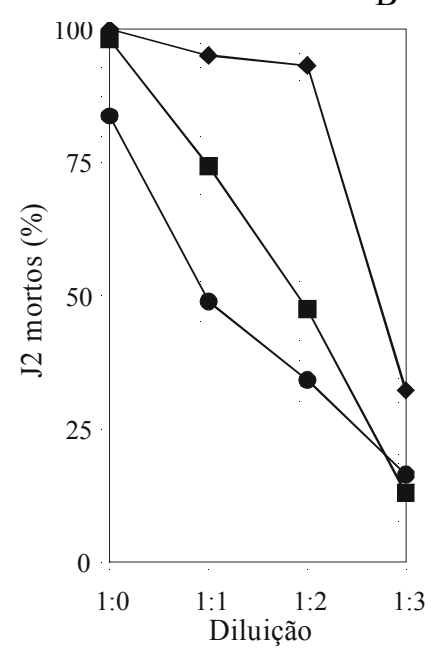

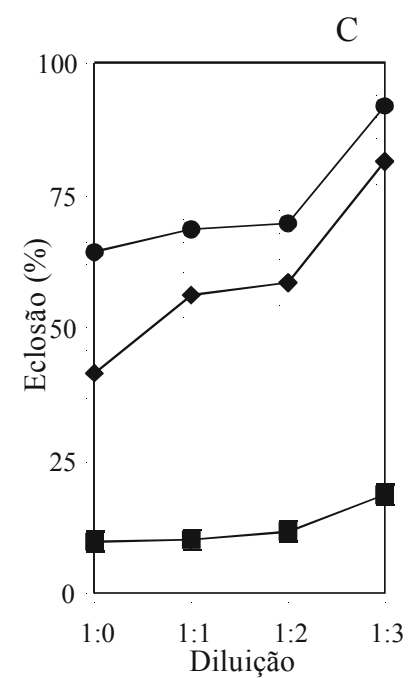

FIG. 3 - Efeito da diluição de filtrados de culturas de Cunninghamella elegans, Fusarium moniliforme e de Paecilomyces lilacinus na motilidade (A), mortalidade (B) e eclosão (C) de juvenis do segundo estádio (J2) de Meloidogyne incognita. Barras verticais indicam erros padrões $(n=6)$. 


\section{REFERÊNCIAS BIBLIOGRÁFICAS}

ASCHNER, M. \& KOHN, S. The biology of Harposporium anguillulae. Journal of General Microbiology 19:182189. 1958.

BARRON, G.L. Isolation and maintenance of endoparasitic nematophagous hyphomycetes. Canadian Journal of Botany 47:1899-1902. 1969.

BARRON, G.L. The nematode-destroying fungi. Canadian Biology Publications, Ottawa. 1977.

BARRON, G.L. \& THORN, R.G. Destruction of nematodes by species of Pleurotus. Canadian Journal of Botany 65:774-778. 1987.

BONETI, J.I.S. \& FERRAZ, S. Modificação do método de Hussey e Barker para extração de ovos de Meloidogyne exigua de cafeeiro. Fitopatologia Brasileira 6:553-561. 1981.

CASTRO, A.G. Defensivos agrícolas como um fator ecológico. Jaguariúna. EMBRAPA - CNPDA. Documento, 6. 1989.

CAYROL, J.C., DIJAN, C. \& PIJAROWSKI. L. Study of the nematicidal properties of the culture filtrate of the nematophagous fungus Paecilomyces lilacinus. Revue de Nématologie 12:331-336. 1989.

COOLEN, W.A. \& D'HERDE, C.J. A method for the quantitative extraction of nematodes from plant tissue. Ghent: State Agriculture Research Centre. 1972.

DIJKSTERHIUS, J. Nematode-fungal interactions. (Ph. D. Thesis). Groningen: University of Groningen. 1993.

DOMSCH, K.H., GAMS, W. \& ANDERSON, T. Compendium of soil fungi. London, Academic Press, 1993.

FITTERS P.F.L., BELDER, E. \& BELDER, E.D. DEN. A time lapse technique to study the effect of fungal products on embryogenesis of nematode eggs. Medelingen van de Faculteit Landbowwetenschappen 58:751-756. 1993.

HUSSEY, R.S. \& BARKER, K.R. A comparison of methods for colecting inocula of Meloidogyne spp. including a new technique. Plant Disease Reporter 57:1025-1028. 1973.

JATALA, P., KAELTENBACH, M. \& BOCANGEL, D.A.J. Field application of Paecilomys lilacinus for controlling Meloidogyne incognita on potatoes. Journal of Nematology 12:226-227. 1980 (Abstract).
KLICH, M.A. \& PITT, J.I. A laboratory guide to common Aspergillus species and their teleomorphs. North Ryde: Commonwealth Scientific and Industrial Research Organization Division of Food Processing. 1984.

LIU, X. \& ZHANG, A. Nematode-trapping species of Monacrosporium with special reference to two new species. Mycological Research 8:862-868. 1994.

MANI A. \& SETHI, C.L. Interaction of root-knot nematode Meloidogyne incognita, Fusarium oxysporum f. sp. ciceri and Fusarium solani on chickpea. Indian Journal of Nematology 17:1-6. 1984.

MANKAU, R.. Biocontrol: Fungi as nematode control agents. Journal of Nematology 12:1-4. 1979.

NAVES, R.L. \& CAMPOS, V.P. Ocorrência de fungos predadores de nematóide no sul de Minas Gerais e estudo da capacidade predatória e crescimento in vitro de alguns de seus isolados. Nematologia Brasileira 15:72-76. 1991.

NELSON, P.E., TOUSSOUN, T.A. \& MARASAS, W.F.O. Fusarium species. An illustrated manual for identification. London, Pensylvania State University Press. 1983.

NICOLAY, R. \& SIKORA, R. A. Improved techniques for the detection of nematophagous fungi and their activity against target nematodes. Revue de Nematologie 11:115-116. 1988.

PITT, J.I. A laboratory guide to common Penicillium species. $2^{\mathrm{a}}$ ed. North Ryde: Commonwealth Scientific and Industrial Research Organization Division of Food Processing. 1988.

PORTER, N. \& FOX, F.M. Diversity of microbial productsdiscovery and application. Pesticide Science 39:161168. 1993.

RUBNER, A. Revision of predacious hyphomycetes in the Dactylella-Monacrosporium complex. Studies in Mycology 39:1-134. 1996.

SCOTT, A.J. \& KNOTT, M. Cluster analysis method for grouping means in the analysis of variance. Biometrics 30:507-512. 1974.

TAYLOR, A.L. \& SASSER, J.N. Biology, identification and control of root-knot nematodes (Meloidogyne species). North Carolina: International Meloidogyne Project. 1978.

WARCUP, J.H. The soil-plate method for isolation of fungi from soil. Nature 166:117. 1950. 